\title{
The Accumulation of $O$-Succinylhomoserine by Escherichia coli and Salmonella typhimurium
}

\author{
BY R. J. ROWBURY \\ Department of Botany, University College London
}

(Received 21 April 1964)

\begin{abstract}
SUMMARY
After growth of Escherichia coli strain 7/9, the culture fluid and organisms contained an amino acid which gave a yellow-brown colour with ninhydrin. This compound was shown by comparison with synthetic material to be $O$-succinylhomoserine believed from previous work with this $E$. coli strain to be a methionine precursor. Its accumulation gave proof that it was formed in large amounts by the growing organisms. The formation of $O$ succinylhomoserine occurred equally well in the presence or absence of added homoserine and succinate, but when the succinate was added to growth media, synthesis occurred preferentially from it and the utilization of endogenously-formed succinate was diminished. Without added homoserine the extent of accumulation was such (up to $440 \mathrm{mg}$. $O$-succinylhomoserine/l.) that homoserine synthesis may have been increased above normal to provide the required quantities (up to $240 \mathrm{mg}$./1.) of homoserine. Methionine completely suppressed accumulation of $\boldsymbol{O}$-succinylhomoserine, threonine and lysine had no effect. A cystathionine-requiring mutant of Salmonella typhimurium also accumulated $O$-succinylhomoserine; the amount (20-30 mg./l.) being only about $5 \%$ of that given by $E$. coli, and methionine prevented its accumulation by this organism too. It appears that the methionine synthesis pathway in $S$. typhimurium is very similar to that in $E$. coli and is subject to end-product control.
\end{abstract}

\section{INTRODUCTION}

For many biosynthetic pathways the occurrence of compounds as intermediates has been established or confirmed by their isolation from organisms or their cultures. For example, the role of homoserine as a methionine precursor in Neurospora was shown by its accumulation in a methionine-requiring strain (Fling \& Horowitz, 1951) and the isolation of cystathionine from another methionine-requiring strain of the same organism (Horowitz, 1947) suggested that cystathionine was an intermediate. The accumulation of methionine precursors by Escherichia coli and Salmonella typhimurium has not been reported previously; Harrold (1962) observed cystathionine accumulation by a mutant of Aerobacter aerogenes. During studies with a methionine-requiring strain of $E$. coli it was observed that the organisms accumulated what appeared to be $O$-succinylhomoserine. The functioning of homoserine and succinate in methionine synthesis had been suggested by studies with auxotrophic mutants of $E$. coli (Davis, 1955; Davis et al. 1959) and $O$-succinylhomoserine had been shown as an intermediate of cystathionine synthesis by experiments with cell-free extracts (Rowbury, 1962b, $c$; Rowbury \& Woods, 1964). This accumulation was studied to obtain conclusive evidence that $O$-succinylhomoserine is a normal intermediate of methionine synthesis in $\boldsymbol{E}$. coli. 
The growth characteristics of methionineless mutants of Salmonella typhimurium suggest that the synthesis pathway is similar to that in Escherichia coli (Smith, 1961), with homoserine, cystathionine and homocysteine as intermediates. Cystathionine-requiring mutants of this organism were therefore screened to ascertain whether accumulation of $O$-succinylhomoserine occurred.

\section{METHODS}

\section{Organisms}

The organisms employed were all auxotrophic strains of Escherichia coli and Salmonella typhimurium and were maintained on slopes of Oxoid nutrient agar, subcultured monthly and stored at $4^{\circ}$ after incubation for $18 \mathrm{hr}$. The strains used were $E$. coli strains $122 / 33,7 / 9,2 / 2,26 / 18$ and B 184 and $S$. typhimurium strains А 15 and в 23. Strains 122/33, 7/9, 2/2, A 15 and в 23 all grew on minimal medium supplemented with methionine or homocysteine or cystathionine. Strain 26/18 required homocysteine or methionine for growth while strain в 184 required homoserine or methionine plus threonine for growth. Strains 26/18, 7/9 and 2/2 were isolated from strain 518, strain 122/33 was isolated from strain $w$ by $\operatorname{Dr} B$. D. Davis and strain B 184 which derived from strain в was provided by Dr J. Gots. The two strains of S. typhimurium were obtained from Dr D. A. Smith.

\section{Growth of organisms and preparation of enzymic extracts}

The medium used was the glucose, lactate and salts medium (GL) of Guest, Helleiner, Cross \& Woods (1960). The medium was supplemented with DL-homoserine $(0.3 \mathrm{mM})$ for strain $B 184$ and with DL-homocysteine $(0.25 \mathrm{~mm})$ for the other strains. Organisms were harvested and enzymic extracts prepared as described previously (Rowbury \& Woods, 1961). The heated extract of organisms from strain $7 / 9$ was prepared by incubating bacteria (equiv. $50 \mathrm{mg}$. dry-wt. $/ \mathrm{ml}$.) in water at $100^{\circ}$ for $10 \mathrm{~min}$. The supernatant fluid! after centrifugation $(10,000 \mathrm{~g}$ for $30 \mathrm{~min}$.) was used as the heated extract.

The assay of $\mathrm{O}$-succinylhomoserine. The cystathionine formed from the compound on incubation in solution $\boldsymbol{A}$ was measured. Solution $\boldsymbol{A}$ contained (in phosphate buffer $\mathrm{pH} 7 \cdot 5,133 \mathrm{~mm}$ ) cysteine $(10 \mu \mathrm{mole})$, ATP (10 $\mu$ mole), glucose (20 $\mu$ mole) and extract from strain $2 / 2$ ( 5 mg. protein). The cystathionine formed was assayed microbiologically with Escherichia coli strain 122/33 as described previously (Rowbury \& Woods, 1964). For comparison purposes samples of synthetic $O$-succinylhomoserine and the compound formed by enzymic extracts were used. The synthetic compound was formed from homoserine and succinic anhydride as described by Rowbury \& Woods (1964). The biologically formed $O$-succinylhomoserine was made by incubation of DL-homoserine (15 $\mu$ mole), sodium succinate (150 $\mu$ mole), ATP (15 $\mu$ mole), glucose $(20 \mu$ mole $)$ and coenzyme A (0.02 $\mu$ mole) with extract from

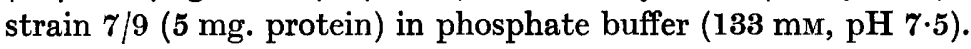

\section{Chromatography}

Paper chromatograms were run in $n$-butanol + propionic acid + water $(47+22+31)$ descending for $16 \mathrm{hr}$. Amino acids were detected by spraying with ninhydrin (0.2\% in $n$-butanol saturated with water). In certain experiments the chromatograms were 
scanned for isotope directly by using the Geiger-Müller tube of a Ratemeter Unit (type 1355 B, Fleming Radio Developments Ltd.).

The $O$-succinyl derivative of homoserine was also chromatographed on columns of Dowex-1 resin. Samples were applied to $18 \times 0.9 \mathrm{~cm}$. columns of resin $(\times 8,200-400$ mesh, acetate form) in aqueous solution. Elution was with water $(15 \mathrm{ml}$.) then with $0 \cdot 2 \mathrm{~N}$-acetic acid. Biological activity of the fractions was assayed with strain $2 / 2$ extract after evaporation to dryness to remove acetic acid. Amino nitrogen in the eluent was assayed with a modified ninhydrin reagent (Moore \& Stein, 1954). In certain cases the radioactivity of the eluent was assessed by spotting samples on uniform ground glass dises and counting at infinite thinness with a Scaler $\mathbf{1 7 0 0}$ (Isotope Developments Ltd.) for 1000 sec. Corrections were made for background activity.

\section{Chemicals}

The sources of most of the special chemicals used were as described previously (Rowbury \& Woods, 1964). ${ }^{14} \mathrm{C}_{2,3}$-succinate was obtained from the Radiochemical Centre, Amersham.

\section{RESULTS}

The isolation of $O$-succinylhomoserine from Escherichia coli strain 7/9

Escherichia coli strain 7/9 (a strain unable to form cystathionine) was previously used for studies on the synthesis of $O$-succinylhomoserine by enzymic extracts (Rowbury, 1962 $a, b$; Rowbury \& Woods, 1964) as it lacks the ability to condense cysteine with $O$-succinylhomoserine. In the present work the organisms were grown with limiting amounts of homocysteine $(0.25 \mathrm{mM}, \mathrm{DL})$ as methionine was known to prevent synthesis of $O$-succinylhomoserine (Rowbury, 1962 $a$ ) and, after harvesting, the growth medium was concentrated and a heated extract prepared from the organisms. The medium and heated extract both showed a ninhydrin positive spot at $\boldsymbol{R}_{\boldsymbol{F}} \mathbf{0 . 3 5}$ when chromatographed on paper in $n$-butanol + propionic acid + water. The compound gave a yellow brown colour (which later turned purple) on heating with ninhydrin. Both synthetic and biologically formed $O$-succinylhomoserine ran at $\boldsymbol{R}_{\boldsymbol{F}} \mathbf{0 . 3 5}$ in this solvent and the yellow brown colour with ninhydrin was characteristic of this compound.

The substance present in the medium and organisms gave rise to cystathionine when incubated with ultrasonic extract from strain 2/2 or strain 26/18. As with synthetic $O$-succinylhomoserine and that formed by enzymic extracts, cysteine was required for cystathionine formation, but homoserine and succinate were not (Table 1). Although extracts from strain $2 / 2$ made cystathionine from the accumulated compound, the growth of strain 2/2 was not stimulated by it (as a replacement for cystathionine) and hence cross-feeding did not occur between strains 7/9 and 2/2. Thus a sample of the accumulated compound ( $1 \mu \mathrm{mole} / \mathrm{ml}$.) had less that $3 \%$ of the growth promoting activity of cystathionine for strain $2 / 2$.

Very large amounts of the cystathionine precursor were present in the growth medium of strain $7 / 9$ but lesser amounts in the organisms (Table 2). During the early stages of this work, homoserine and succinate were added to growth media but this was later shown to be unnecessary (Table 2) as these compounds did not increase the accumulation. Under optimal conditions up to $2000 \mu$ mole of the precursor were formed per litre of medium. 
Other strains of Escherichia coli were tested to ascertain whether the cystathionine precursor accumulated. Three strains were tested, one (strain 2/2) unable to form cystathionine, a second which is unable to carry out a step before cystathionine formation (strain B 184, a homoserine requirer, lacking homoserine dehydrogenase) and a third strain lacking the cystathionase enzyme (strain 26/18). None of these strains accumulated the precursor.

Table 1. The synthesis of cystathionine from the accumulated compound and from synthetic $\mathrm{O}$-succinyl homoserine

Organisms (Escherichia coli strain $7 / 9$ ) were harvested from medium GL +homocysteine, the culture fluid concentrated by evaporation in vacuo, and samples incubated in $3 \mathrm{ml}$. of solution $A$ at $37^{\circ}$ with the stated omissions and additions. The $O$-succinylhomoserine was a synthetic sample purified on Dowex-1 resin.

\begin{tabular}{lcc}
\multicolumn{1}{c}{ Additions } & $\begin{array}{c}\text { From the accumulated } \\
\text { compound }\end{array}$ & $\begin{array}{c}\text { Cystathionine formed } \\
(\mu \mathrm{mmole} / \mathrm{mg} \text {. protein })\end{array}$ \\
homoserine (2 mM)
\end{tabular}

Table 2. The extent of the accumulation of the cystathionine precursor in Escherichia coli strain $7 / 9$

Organisms were grown on medium GL+homocysteine $(0.3 \mathrm{~mm})$ with the stated additions for $18 \mathrm{hr}$ at $37^{\circ}$. In all cases the medium was concentrated in vacuo, and in one case a heated extract of the organisms was prepared. The precursor was assayed by cystathionine formation in solution $\mathbf{A}$.

\begin{tabular}{lcc} 
Additions & \multicolumn{2}{c}{$\begin{array}{c}\text { Cystathionine precursor } \\
(\mu \text { mole/l. culture })\end{array}$} \\
None & - & In medium \\
Homoserine $(2 \mathrm{mM})+$ & 60 & 1640 \\
succinate $(20 \mathrm{~mm})$ & & 1400 \\
Homoserine $(2 \mathrm{mM})$ & - & 1480 \\
Succinate $(20 \mathrm{~mm})$ & - & 1550
\end{tabular}

The purification and properties of the accumulated compound

Homoserine and other neutral amino acids can be readily removed from samples of $O$-succinylhomoserine by chromatography on Dowex-1 resin (Rowbury \& Woods, 1964) as $O$-succinylhomoserine (due to its slight acidity) is retained by the resin when a neutral solution is applied, but is eluted with $0.2 \mathrm{~N}$ acetic acid. The cystathionine precursor accumulated by strain $7 / 9$ was also retained by the resin and on addition of $0.2 \mathrm{~N}$ acetic acid was eluted in the same position as $O$-succinylhomoserine (Fig. 1).

A purified sample of the accumulated compound was very unstable to mild alkali but more stable in mild acid solution (Fig. 2); extreme instability to alkali was a characteristic of $O$-succinylhomoserine (Rowbury, $1962 b$; Rowbury \& Woods, 1964), and the two compounds behaved very similarly over the range pH 2-11 (Fig. 2). 
Although the precursor was stable to treatment with mild acid, more vigorous conditions ( $\mathrm{N}-\mathrm{HCl}$ for $2 \mathrm{hr}$ at $100^{\circ}$ ) destroyed its biological activity and paper chromatography showed the presence of homoserine in the hydrolysate.

\section{The presence of succinate in the cystathionine precursor}

When studying $O$-succinylhomoserine formation by enzymic extracts, the incorporation of succinate was shown by using ${ }^{14} \mathrm{C}$-succinate (Rowbury, 1962b). To demonstrate the presence of a succinate residue in the accumulated compound,

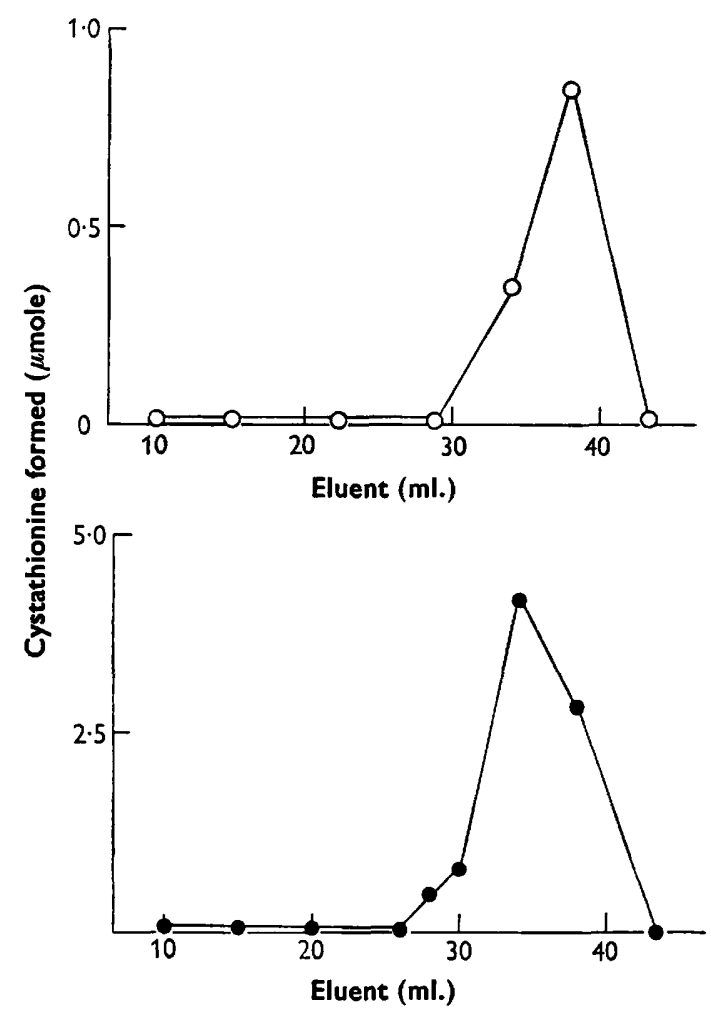

Fig. 1. Comparison of the behaviour of the accumulated cystathionine precursor and synthetic $O$-succinylhomoserine on Dowex-1 resin. Samples of the precursor and of synthetic compound were applied to columns of Dowex-1 resin and eluted as described in Methods. Biological activity was assayed by incubation in solution A. $O$, synthetic $O$-succinyl homoserine; $O$, cystathionine precursor.

organisms were grown on medium GL with limiting homocysteine and with ${ }^{14} \mathrm{C}$ succinate added. When the concentrated growth medium was chromatographed on Dowex-1 resin, two peaks of isotope were observed (Fig. 3). The first peak probably represented incorporation into neutral amino acids; the second peak was due to incorporation of succinate into the precursor as it coincided with the peak of biological activity (i.e cystathionine formation when incubated with strain $2 / 2$ extract in solution $A$ ). On paper chromatography of a sample from this second peak, all the isotope ran at $\boldsymbol{R}_{F} \mathbf{0 \cdot 3 5}$ (in $n$-butanol + propionic acid + water) coinciding with the spot of ninhydrin-positive material. 
An appreciable amount of the added ${ }^{14} \mathrm{C}$-succinate was incorporated into the precursor even when unlabelled succinate was added to the growth medium (Table 3). When $10 \mathrm{~mm}$ succinate was added, $10 \%$ of the isotope was incorporated into the precursor and with $25 \mathrm{~mm}$ succinate present, $5 \%$ was incorporated. When the amount of the precursor (in terms of biological activity) was compared to the extent of isotope incorporation it was apparent that most of the accumulated compound had arisen from added succinate rather than from endogenously formed succinate

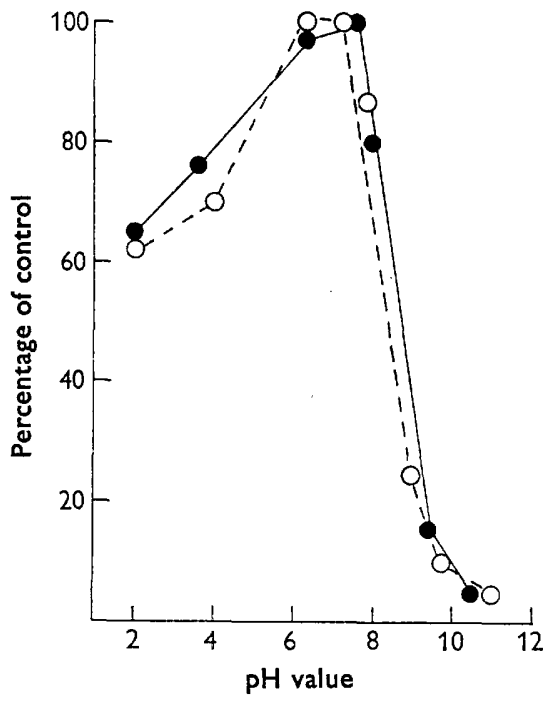

Fig. 2

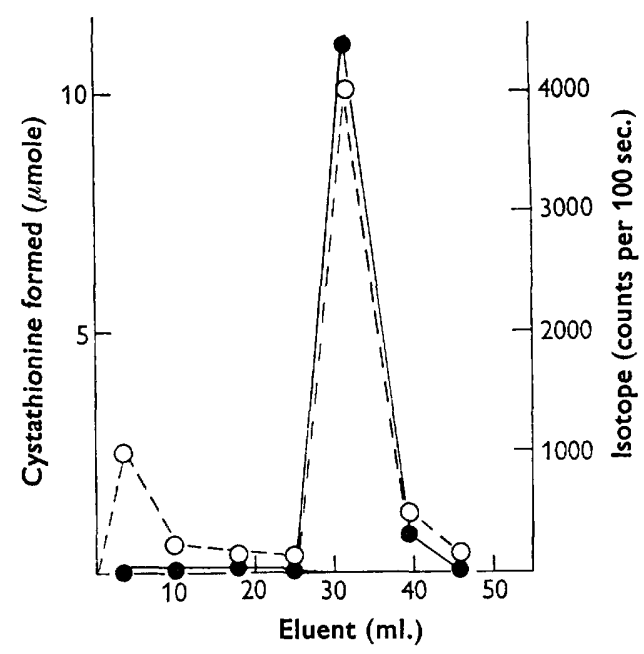

Fig. 3

Fig. 2. The effect of mild acid and alkali on accumulated precursor. Samples of the accumulated compound and synthetic $O$-succinylhomoserine were incubated at the stated $\mathrm{pH}$ value for $5 \mathrm{~min}$. at $37^{\circ}$, then readjusted to $\mathrm{pH} 7.5$ and incubated in solution A for $4 \mathrm{hr}$. Cystathionine formed was assayed with Escherichia coli strain 122/33. synthetic $O$-succinylhomoserine; $O$, the accumulated cystathionine precursor.

Fig. 3. The presence of a succinate residue in the accumulated cystathionine precursor. Organisms of Escherichia coli strain 7/9 were grown on medium GL with added ${ }^{14} \mathrm{C}_{2,3^{-}}$ succinate and the medium chromatographed on Dowex-1 resin as described in Methods. Biological activity of the fractions was measured in solution $\boldsymbol{A}$ and radioactivity assessed as described in Methods. O, biological activity; $\bigcirc$, radioactivity.

Table 3. The incorporation of exogenous succinate into the cystathionine precursor

Organisms (Escherichia coli strain $7 / 9$ ) were grown on medium GL + homocysteine with the stated concentration of succinate as well as ${ }^{14} \mathrm{C}$-succinate $(5 \mu \mathrm{c}$. total). The culture fluid (10 ml. total) was concentrated in vacuo and applied to a column of Dowex-1 resin. Samples of the eluate were assayed for isotope as described in Methods and for biological activity by incubation in solution $\mathbf{A}$.

Cystathionine precursor formed

\section{Succinate in medium (mM)}

10 $\mathbf{2 5}$

$\overbrace{\begin{array}{c}\text { A. Total } \\ (\mu \text { mole })\end{array}}^{\begin{array}{c}\text { From }{ }^{14} \mathrm{C}- \\ \text { succinate } \\ (\mu \text { mole })\end{array}} \begin{gathered}\text { \% from } \\ { }^{14} \mathrm{C} \text {-succinate }\end{gathered}$

$11 \cdot 2$

12.0
82

92 
(Table 3). When $25 \mathrm{~mm}$ succinate was present $92 \%$ of the precursor had arisen from exogenous succinate and only $8 \%$ from endogenous sources.

\section{The effect of methionine on the accumulation of the cystathionine precursor}

Accumulations generally occur only after the growth of cultures has ceased and when any added metabolite has been exhausted (Gots, 1957). For this reason the experiments described above were with organisms grown with limited quantities of homocysteine. When DL-methionine (3.3 mM) was added to the medium, it was observed by paper chromatography that the cystathionine precursor was not accumulated; this was confirmed by chromatography on Dowex-1 resin columns. The precursor was separated from methionine (which would interfere with the bioassay of cystathionine) and assayed for cystathionine synthesis in solution $A$ or with ninhydrin (Fig. 4). Methionine reduced the accumulation of the precursor to less than $2 \%$ although lysine and threonine (which have partly common biosynthetic pathways with methionine) did not prevent the accumulation when added to media (Table 4).

\section{Table 4. The effect of methionine, threonine and lysine on the accumulation of the cystathionine precursor}

Organisms (Escherichia coli strain $7 / 9)$ were grown on medium GL + homocysteine
with the stated additions for $18 \mathrm{hr}$ at $37^{\circ}$, and the culture fluid ( $50 \mathrm{ml}$.) concentrated in
vacuo. Where methionine had been added, the medium was chromatographed on Dowex-1
resin and the appropriate fractions assayed in solution A for the cystathionine precursor.
The other samples were assayed directly.
\[ \begin{array}{cc}\text { Cystathionine } \\ \text { precursor } \\ (\mu \text { mole total) }\end{array} \]
\[ \begin{array}{cc}\text { Addition } & 147 \\ \text { None } & 2 \\ \text { DL-methionine }(3 \cdot 3 \mathrm{~mm}) & 147 \\ \text { DL-threonine (3.3 } \mathrm{mM}) & 144 \\ \text { DL-lysine }(3 \cdot 3 \mathrm{~mm}) & \end{array} \]

The accumulation of $\mathrm{O}$-succinylhomoserine by Salmonella typhimurium

Smith (1961) has described a series of methionineless mutants of Salmonella typhimurium, two of which (strains A 15 and B 23) appeared unable to form cystathionine. The strains were tested for accumulations by growing them with limiting amounts of homocysteine and chromatographing the concentrated medium on paper chromatograms. The medium from strain $\mathrm{B} 23$ contained a compound which ran at $R_{F} \mathbf{0 . 3 5}$ in $n$-butanol-propionic acid-water and gave a yellow-brown colour with ninhydrin suggesting that it was $O$-succinylhomoserine. Strain a 15 did not show any marked accumulation of amino acids. The extent of accumulation by strain в 23, when assayed by using extract from Escherichia coli strain 2/2, proved to be about 100-150 $\mu$ mole/l. as compared with up to $2000 \mu \mathrm{mole} / \mathrm{l}$. for $E$. coli strain $7 / 9$.

To confirm that the compound present in the culture fluid from strain в 23 was $O$-succinylhomoserine, organisms were grown on medium supplemented with ${ }^{14} \mathrm{C}$ succinate, the fluid chromatographed on Dowex-1 resin and samples of the eluate tested for radioactivity. A labelled compound was eluted in the same place as $\boldsymbol{O}$ succinylhomoserine (Fig. 5), and when a sample was run on a paper chromatogram 
the isotope ran with synthetic $O$-succinylhomoserine. The accumulation of $O$-succinylhomoserine by strain B 23 (like that with strain 7/9) was completely abolished when the organisms were grown with methionine. The absence of the compound was noted when culture fluids were chromatographed on paper; this was confirmed by using ${ }^{14} \mathrm{C}$-succinate. DL-methionine (3.3 $\mathrm{mm}$ ) decreased the incorporation of label from succinate to less than $3 \%$ (Fig. 5), although growth of the organisms was greater with added methionine.

Synthetic $O$-succinylhomoserine did not support the growth of strains A 15 or B 23, neither did culture fluid from strain B 23 stimulate the growth of strain A 15 . Thus a Seitz-filtered sample of $O$-succinylhomoserine which contained $0.9 \mu$ moles/ ml. (assayed with extract from strain $2 / 2$ ) had less than $2 \%$ of the activity of the same amount of cystathionine for strains $\mathrm{A} 15$ and $\mathrm{B} 23$.

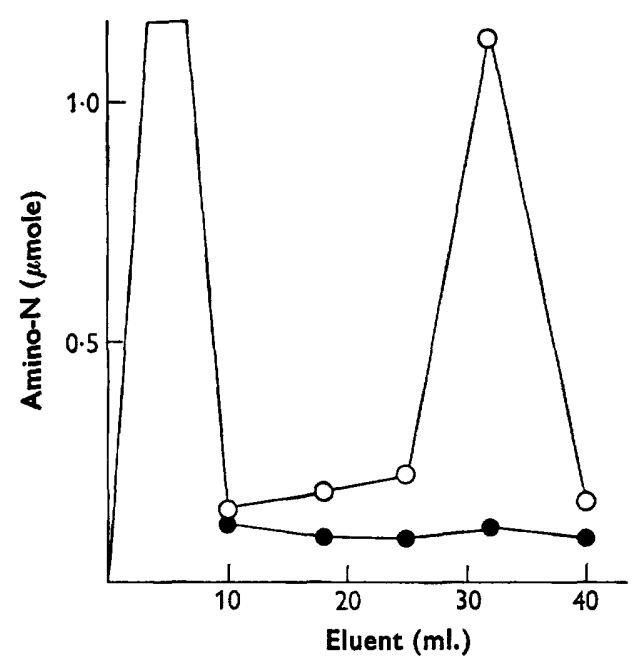

Fig. 4

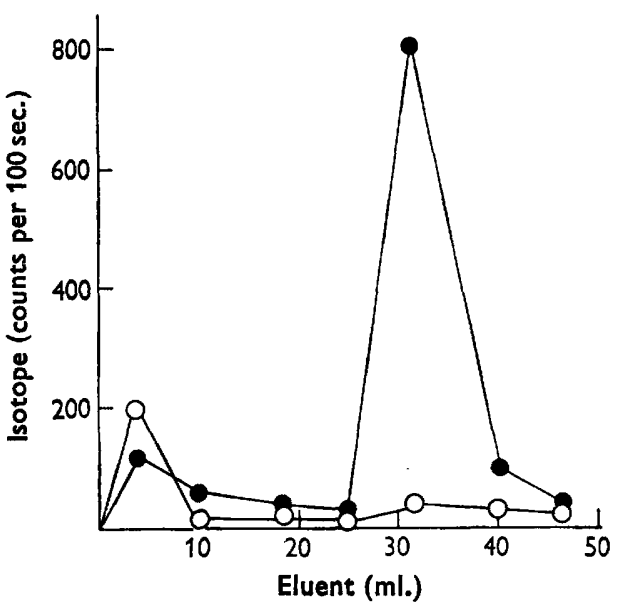

Fig. 5

Fig. 4. The effect of methionine on the accumulation of the cystathionine precursor in the medium of Escherichia coli strain 7/9. Organisms were grown in medium GL with methionine (8.3 mM) present (O) or absent (O). The media were chromatographed on Dowex-1 resin and amino- $N$ assayed with the modified ninhydrin reagent.

Fig. 5. Inhibition by methionine of the accumulation of the cystathionine precursor in the medium of Salmonella typhimurium strain B23. Organisms were harvested from medium GL supplemented with ${ }^{14} \mathrm{C}$-succinate with methionine (3.8 $\left.\mathrm{mM}\right)$ present $(O)$ or absent (O) and the medium chromatographed on Dowex-1 resin. Radioactivity was measured as described in Methods.

\section{DISCUSSION}

Analysis of the compound accumulated by Escherichia coli $7 / 9$ showed it contained homoserine and succinate and the reaction with ninhydrin demonstrated the presence of a free amino group. $O$-acyl derivatives of hydroxyamino acids are very unstable to alkali (Sakami \& Toennies, 1942) so that $O$-succinylhomoserine was the most probable structure; the behaviour of the compound as a weak acid on Dowex-1 resin was compatible with this structure. Finally, the identity of the compound 
with $O$-succinylhomoserine was shown by comparison with the synthetic compound: the two substances behaved alike chromatographically and both gave rise to cystathionine when incubated with cysteine and extract from strain $2 / 2$.

The accumulated $\boldsymbol{O}$-succinylhomoserine was found predominantly in the culture fluid (Table 2) and, since the lysis of dying organisms could only supply a small part of this, the compound must be able to leak out of growing organisms. This is of particular interest since $O$-succinylhomoserine does not support the growth of Escherichia coli strain $2 / 2$ although this strain has enzymic mechanisms for the synthesis of cystathionine from it. Although it is possible that the compound is destroyed after entry into the organisms, it seems more likely that this strain is not able to absorb it at all, although the compound is able to leak out of $E$. coli strain 7/9. The accumulation of $O$-succinylhomoserine by cultures of $E$. coli strain $7 / 9$ was not increased by exogenous homoserine or succinate, so that these organisms were able to supply the extra amounts of these compounds then required. The supply of succinate could be readily achieved by increasing the supply of Krebs cycle intermediates, but the extra homoserine synthesis (up to $240 \mathrm{mg}$./1.) may require increased amounts of the synthesizing enzymes. Although the accumulation of $O$ succinylhomoserine was not increased by supplementing the medium with succinate, as exogenous succinate was very efficiently used for synthesis of $O$-succinylhomoserine, the succinate must be readily equilibrated with the cellular pool used for $O$-succinylhomoserine synthesis. Since succinyl-coenzyme $A$ is the active form of succinate used in the reaction (Rowbury \& Woods, 1964), the endogenous succinate probably arises from $\alpha$-oxoglutarate. This is supported by the observation that an auxotroph of $\boldsymbol{E}$. coli which lacks the ability to convert $\alpha$-oxoglutarate to succinyl-coA requires methionine for growth (Davis et al. 1959). Whether the added succinate exerts any effect on succinate synthesis is not certain.

Previously the evidence for the functioning of $\boldsymbol{O}$-succinylhomoserine as a methionine precursor in Escherichia coli had been based on its synthesis by extracts of $\boldsymbol{E}$. coli $7 / 9$ from homoserine and succinyl-coenzyme $A$ (activity being absent from E. coli $2 / 2$ ) and its conversion to cystathionine by $E$. coli 2/2. (Rowbury, 1962b; Rowbury \& Woods, 1964). Since strain 7/9 lacks the latter activity, the accumulation of $O$-succinylhomoserine is readily explicable and provides conclusive evidence that the compound is a bona fide methionine precursor. The failure toisolate the precursor from the culture fluid of three other methionine-requiring strains of $\boldsymbol{E}$. coli confirms that the accumulation is a specific result of the metabolic lesion of $E$. coli 7/9. The abolition of accumulation by growth with methionine also implies that the accumulated compound plays an essential role in methionine synthesis and confirms evidence obtained at the enzymic level (Rowbury, 1962a) that methionine controls this stage of synthesis. The excessive production of $\boldsymbol{O}$-succinyl homoserine was therefore due to exhaustion of the limited supply of homocysteine.

Smith (1961) showed that certain methionine-requiring mutants of Salmonella typhimurium responded to cystathionine, and since wild type $S$. typhimurium has cystathionase activity (Rowbury, 1962c) cystathionine seemed to be a precursor of methionine in that organism. This is supported by the isolation of $O$-succinylhomoserine from the culture fluid of the cystathionine-requiring $S$. typhimurium strain B 23. The observation that methionine greatly decreased the accumulation supports the view that the compound is a normal intermediate. 
The extent of the accumulations of $\boldsymbol{O}$-succinylhomoserine in Escherichia coli and Salmonella typhimurium deserves some comment. S. typhimurium strain в 23 may be more sensitive to the presence of traces of methionine or the enzymes of methionine synthesis may be less active, since the accumulation was much less in this organism. The accumulation of $O$-succinylhomoserine in $E$. coli strain $7 / 9$ must require marked changes in metabolism to provide the amounts of substrates and energy required as the extent of the accumulation greatly exceeds that of methionine precursors in Neurospora crassa. Thus one cystathionine-requiring mutant of N. crassa (Fischer, 1957) accumulated up to $2 \cdot 4 \%$ cystathionine (of dry wt. organism), while another accumulated $1.5 \mathrm{~g}$. threonine/kg. wet weight (probably about $3 \%$ dry wt.) and lesser amounts of homoserine (Fling \& Horowitz, 1951) whereas the accumulation of $O$-succinylhomoserine by $E$. coli strain $7 / 9$ was up to $40 \%$ of the dry weight.

\section{REFERENCES}

Davis, B. D. (1955). Intermediates in amino acid biosynthesis. Advanc. Enzymol. 16, 247.

Davis, B. D., Kornberg, H. L., Nagler, A., Mrluer, P. \& Mingioli, E. (1959). Formation and functions of succinate in Escherichia coli. Fed. Proc. 18, 211.

Fischer, G. A. (1957). The cleavage and synthesis of cystathionine in wild type and mutant strains of Neurospora crassa. Biochim. biophys. Acta, 25, 50.

Fuing, M. \& Horowitz, N. H. (1951). Threonine and homoserine in extracts of a methionineless mutant of Neurospora. J. biol. Chem. 190, 277.

Gors, J. (1957). Purine metabolism in bacteria. V. Feedback Inhibition. J. biol. Chem. 228, 57.

Guest, J. R., Heumeiner, C. W., Cross, M. J. \& Woods, D. D. (1960). Cobalamin and the synthesis of methionine by ultrasonic extracts of Escherichia coli. Biochem. J. 76, 396.

HARROLD, F. M. (1962). Accumulation of cystathionine in a homocysteine requiring mutant of Aerobacter aerogenes. J. Bact. 84, 382.

Horowitz, N. H. (1947). Methionine synthesis in Neurospora. The isolation of cystathionine. J. biol. Chem. 171, 255.

Moore, S. \& SteIn, W. H. (1954). A modified reagent for the photometric determination of amino acids and related compounds. J. biol. Chem. 211, 907.

Rowbury, R. J. (1962a). Control of cystathionine formation in Escherichia coli by methionine. Biochem. J. 82, 24 P.

Rowbury, R. J. (1962b). A succinyl derivative of homoserine as a precursor of methionine in Escherichia coli. J. gen. Microbiol. 28, $5 P$.

ROWBURY, R. J. (1962c). Factors influencing the control of methionine synthesis in microorganisms. D. Phil. Thesis: Oxford University.

Rowbury, R. J. \& Woods, D. D. (1961). Further studies on the repression of methionine synthesis in Escherichia coli. J. gen. Microbiol. 24, 129.

Rowbury, R. J. \& Woods, D. D. (1964). $O$-Succinyl homoserine as an intermediate in the synthesis of cystathionine by Escherichia coli. J. gen. Microbiol. (in the Press).

SAKami, W. \& Toennies, G. (1942). The investigation of amino acid reactions by methods of non-aqueous titrimetry: II. Differential acetylation of hydroxyl groups and a method for the preparation of $O$-acetyl derivatives of hydroxy amino acids. J. biol. Chem. 144, 203.

SмIтH, D. A. (1961). Some aspects of the genetics of methionineless mutants of Salmonella typhimurium. J. gen. Microbiol. 24, 335. 